\title{
Atrial septal defect closure in a midget toy poodle
}

\author{
Keisuke Sugimoto ${ }^{1 *}$, Yohei Mochizuki ${ }^{1}$, Teppei Kanda ${ }^{1}$, Akihiro Ohnishi $^{1}$, Masahiro Miyabe ${ }^{1}$, Yuko Wada ${ }^{1}$, \\ Masaki Kochi ${ }^{2}$ and Takuma Aoki ${ }^{3}$ \\ ${ }^{1}$ Department of Veterinary Medicine, Okayama University of Science, Imabari, Ehime 794-8555, Japan \\ ${ }^{2}$ Division of Cardiology, Matsubara Animal Hospital, Matsubara, Osaka 580-0044, Japan \\ ${ }^{3}$ Department of Veterinary Medicine, Azabu University, Sagamihara, Kanagawa 252-5201, Japan
}

\begin{abstract}
Background: Atrial septal defect (ASD) is a rare congenital cardiac disease, and there have been no reports about the treatment of ASD in midget breed dogs.

Case Description: A 7-month-old female toy poodle weighing $1.4 \mathrm{~kg}$ presented with cardiac enlargement. Echocardiography revealed a secundum-type ASD, right ventricular and atrial enlargement, and pulmonary hypertension. Blood flow through the ASD exhibited left-to-right shunting. The dog underwent ASD closure through a hybrid approach, in conjunction with catheter techniques and thoracotomy. Ten months after treatment, cardiac enlargement and pulmonary hypertension were improved.
\end{abstract}

Conclusion: Even in midget dog breeds, ASD can be corrected through a hybrid approach.

Keywords: Canine, Cardiology, Catheter, Congestive heart failure, Small breed.

\section{Introduction}

Atrial septal defect (ASD) is characterized by communication between the two atria due to a defect in the interatrial septum (Chetboul et al., 2006), and it is a rare congenital cardiovascular defect in dogs (Schrope, 2015). The long-term prognosis of dogs with an isolated small-sized defect is usually good. Conversely, in dogs with large-sized defects, a significant left-to-right shunt results in the right ventricular (RV) volume overload and increased pulmonary blood flow (Guglielmini et al., 2002). This increase results in pulmonary hypertension or right heart failure with associated exercise intolerance (Guglielmini et al., 2002). Thus, treatment is required in dogs with large-sized defects.

Catheter-based techniques and open-heart procedures have been employed to close large-sized defects in dogs, and the successful treatments have been reported in large dog breeds (Eyster et al., 1976; Gordon et al., 2009; Sanders et al., 2005; Shelden et al., 2017). In contrast, there are no reports of such treatments being administered in small and midget dog breeds, as the size is too small to perform these procedures (Pelosi et al., 2013).

The hybrid approach incorporates catheter techniques into cardiac surgery. Hybrid approaches in cases of cardiovascular disease are a treatment option in children weighing less than $5 \mathrm{~kg}$ when vascular access is limited by small patient size, precluding device delivery, and cardiopulmonary bypass is not required (Amin et al., 2008), and they have recently been used in human medicine (Carminati et al., 2007; Doshi et al., 2018; Driver and Mangrum, 2015; Ohnishi, 2012; Li et al.,
2013). In veterinary medicine, the hybrid approach has also been applied in the treatment of canine ASD (Uemura et al., 2018; Zani et al., 2016) but for dogs larger than $5 \mathrm{~kg}$. Here, we describe a case of ASD closure in a midget toy poodle using a hybrid approach combined with catheter techniques and thoracotomy.

\section{Case Details}

A 7-month-old female toy poodle weighing $1.4 \mathrm{~kg}$ was referred to the Okayama University of Science Veterinary Teaching Hospital with cardiac dilatation. The dog was maldeveloped. No cardiac murmur was detectable through thoracic auscultation, and there were no respiratory abnormalities. Complete blood count and serum biochemical test results were within reference ranges. Thoracic radiography revealed a right-sided cardiomegaly, pulmonary overcirculation, and main pulmonary artery enlargement without any tracheal, bronchial, or pulmonary abnormalities or lesions (Fig. 1a and b). Electrocardiography revealed a right-axis deviation $\left(-150^{\circ}\right)$ as well as a deep S-wave $(-1.5 \mathrm{mV})$ and no arrhythmia. Echocardiography evaluation revealed moderate dilatation and concentric hypertrophy of the RV with ventricular septal flattening, enlargement of the right atrium (RA), and main pulmonary artery enlargement (pulmonary artery-to-aorta ratio: 1.34). A secundum-type ASD was also observed, and the blood flows though the defect exhibited a left-to-right shunt (Fig. 1c-e). The size of the defect was $3.3-4.0 \mathrm{~mm}$, and the pulmonary blood flow/systemic blood flow ratio $(\mathrm{Qp} / \mathrm{Qs})$ as determined through echocardiography was 3.03 . Based on these 

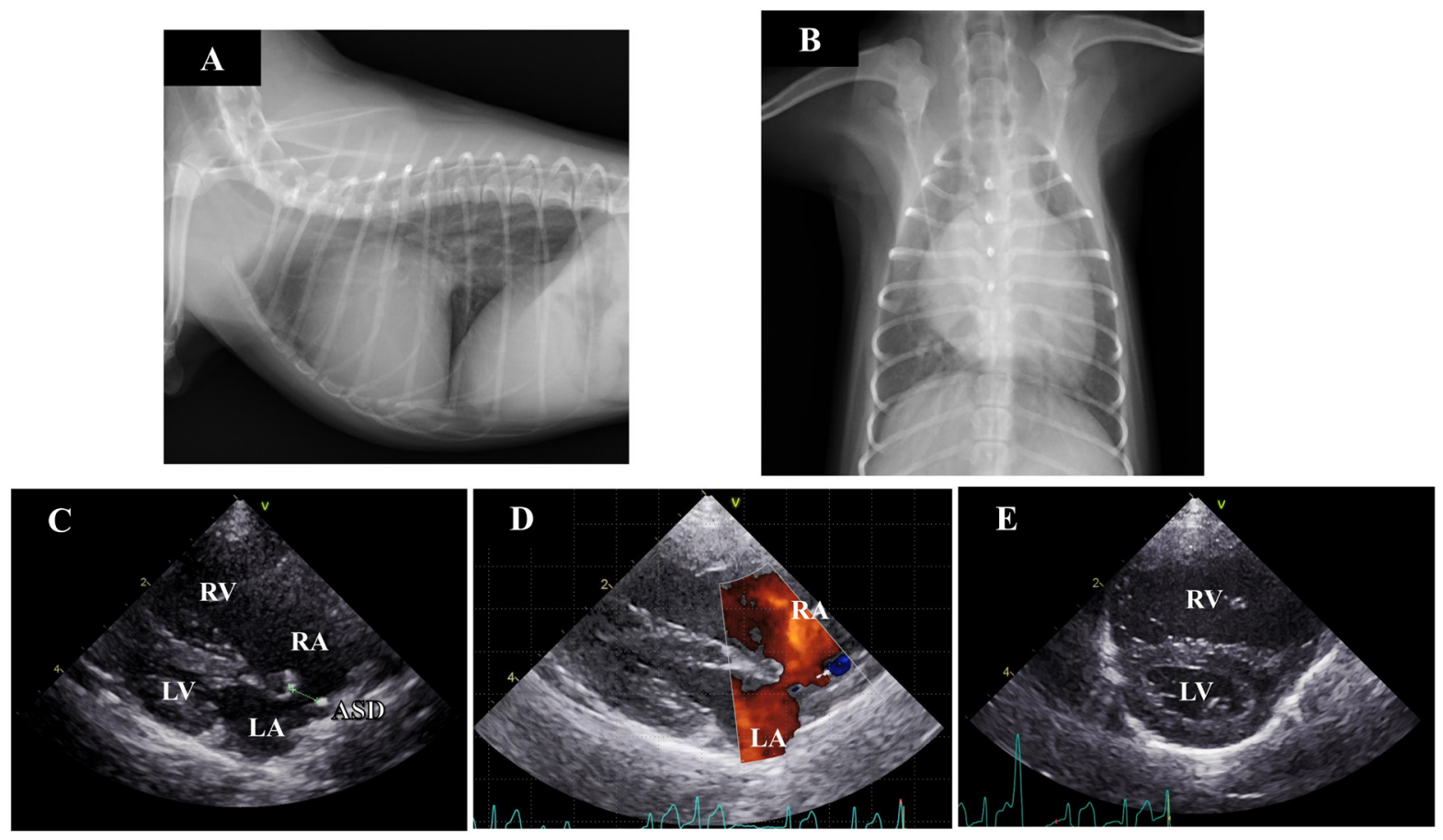

Fig. 1. Thoracic radiography and echocardiography on the first examination. (a) and (b): Thoracic radiography revealed a right-sided cardiomegaly and pulmonary artery enlargement without any abnormality in the respiratory tract. (c)-(e): Echocardiography revealed left-to-right shunting ASD and eccentric and concentric hypertrophy of the right ventricle with ventricular septal flattening. (ASD): atrial septal defect; (LA): left atrium; (LV): left ventricle; (RA): right atrium; (RV): right ventricle.

observations, the isolated secundum-type ASD was diagnosed. Due to the risk of progression of pulmonary hypertension, heart failure, or right-to-left shunt, ASD closure using an Amplatz canine duct occluder was planned.

Preanesthetic medications included fentanyl citrate (3 $\mu \mathrm{g} / \mathrm{kg} \mathrm{IV})$ and robenacoxib (2 $\mathrm{mg} / \mathrm{kg} \mathrm{SC})$. The dog was anesthetized with alfaxalone (3 $\mathrm{mg} / \mathrm{kg}$ IV) and intubated. General anesthesia was maintained with sevoflurane mixed with $100 \% \mathrm{O}_{2}$ and infusion of fentanyl citrate at a constant rate $(10 \mu \mathrm{g} / \mathrm{kg} / \mathrm{h})$, under controlled ventilation. After intubation, intercostal nerve blocks were performed on the incision area with lidocaine, followed by administration of atropine sulfate $(20 \mu \mathrm{g} / \mathrm{kg} \mathrm{IM})$, cefazolin $(30 \mathrm{mg} /$ $\mathrm{kg} \mathrm{IV})$, and vecuronium bromide (0.05 $\mathrm{mg} / \mathrm{kg} \mathrm{IV})$. Heart rate, pulse rate, noninvasive arterial blood pressure, electrocardiography tracings, respiratory rate, tidal volume, minute volume, rectal temperature, arterial oxygen saturation, end-tidal carbon dioxide concentration, and sevoflurane concentration were monitored continuously during the surgery. The dog was restrained in the left lateral recumbent position.

The right thoracotomy was performed in the fifth intercostal space. After thoracotomy, the pericardium was incised above the phrenic nerve. Dilation of the RA and RV was visually confirmed. The size of the defect was $4.2 \mathrm{~mm}$, as determined through trans right atrial echocardiography with a $12-\mathrm{MHz}$ transducer (Vivid E95, GE Healthcare Japan, Tokyo, Japan). A purse-string ligature (Nescosuture 4-0 taper point, Alfresa Pharma Co. LTD, Osaka, Japan) was formed in the right atrial wall, the atrial wall was punctured with scalpel (No. 11, Akiyama Medical MFG. Co. Ltd., Tokyo, Japan), and catheterization was performed under trans right atrial echocardiographic guidance. The $5 \mathrm{Fr}$ catheter (Destination, Terumo Co. LTD, Tokyo, Japan) was advanced across the atrial defect into the left atrium without guide wire. The first disc and part of the waist (4 mm) (Amplatz canine duct occluder, Infiniti Medical LLC, CA, USA) were deployed in the left atrium. The device was seen to go through the ASD however, so it was exchanged with a different size (waist size 6 $\mathrm{mm}$ ). The catheter was advanced again, and the device was deployed gently against the atrial septum. While back tension was maintained on the delivery cable, the catheter was retracted such that the second disc was unsheathed within the RA. After deployment, the device was released, the catheter was removed from the atrium, and both the purse-string sutures (Nescosuture 4-0, Alfresa Pharma Co. Ltd., Osaka, Japan) were tied. The thorax was closed in a routine manner, and the drain catheter (8 Fr Atom Multipurpose Tube, Atom Medical Co. LTD, Tokyo, Japan) was placed. The total anesthesia time was 185 minutes, and the operation time was 132 minutes. Recovery from anesthesia was 
uncomplicated. The dog was administered antibiotics (cefazolin, $30 \mathrm{mg} / \mathrm{kg}$, q 8 hours IV for 3 days, and cefalexin, $15 \mathrm{mg} / \mathrm{kg}$, q 12 hours PO for the next 7 days) after the surgery. Antiplatelet medicine was initiated (aspirin, $0.5 \mathrm{mg} / \mathrm{kg}$, q 24 hours PO for 5 months) after the removal of drain catheter (2 days after surgery). At 2 and 5 days after surgery, red blood cell counts, hematocrit, and hemoglobin content were within the reference ranges and had not noticeably changed compared to their values before surgery. The dog was discharged with instructions to severely limit exercise for 4 weeks and then to limit activity for 3 months.

Five days after the operation, dilatation and concentric hypertrophy of the RV, ventricular septal flattening, pulmonary overcirculation, and main pulmonary artery enlargement were improved (pulmonary artery-toaorta ratio: 1.05) (Fig. 2a-e) although mild residual shunting was observed (Fig. 2d). At 10 months after the operation, the dog had gained weight $(1.74 \mathrm{~kg}), \mathrm{RV}$ enlargement was further improved, and the Qp/Qs had decreased to 1.16 .

\section{Discussion}

This is the first report of a case in which ASD closure using a hybrid approach was successfully performed in a midget dog breed. We used a method that differed from the previous approaches to similar defects in dogs. Gordon et al. (2009) reported the use of transesophageal echocardiography for transatrial ASD closure in a large dog. Uemura et al. (2018) reported the use of transesophageal echocardiography and computed tomography angiography in a dog weighing $6.7 \mathrm{~kg}$. In the present case, the dog was too small for transesophageal echocardiography (Domenech and Oliveira, 2013), so we used transatrial echocardiography for catheterization. Furthermore, we chose a different intercostal entry from that used in the aforementioned reports : repeated assessment through thoracic radiography and transthoracic echocardiography informed our decision to gain access through the fifth intercostal space. Differences in patient size or the degree of cardiac enlargement may influence this choice in such cases.

The method utilized in the current case was problematic in one respect. The device was seen to go through the defect on the first occasion, and the main reason for this was underestimation of the ASD. Transthoracic and transatrial echocardiography was used to assess the size of the ASD. To determine a defect's size, the use of a balloon catheter is recommended (Gordon et al. 2009). This was not done in the present case due to the possibility of perforation or right atrial rupture associated with the use of a balloon catheter. In many cases, ASDs are elliptical (Zanchetta et al., 2003), and this can lead to underestimation of the size of the defect. In humans, ASD cardiac magnetic resonance imaging, cardiac computed tomography angiography, or $3 \mathrm{D}$ echocardiography are recommended to assess defects (Brown and Kanmanthareddy, 2019; Taniguchi et al., 2009; van den Bosch et al., 2006). Although, in
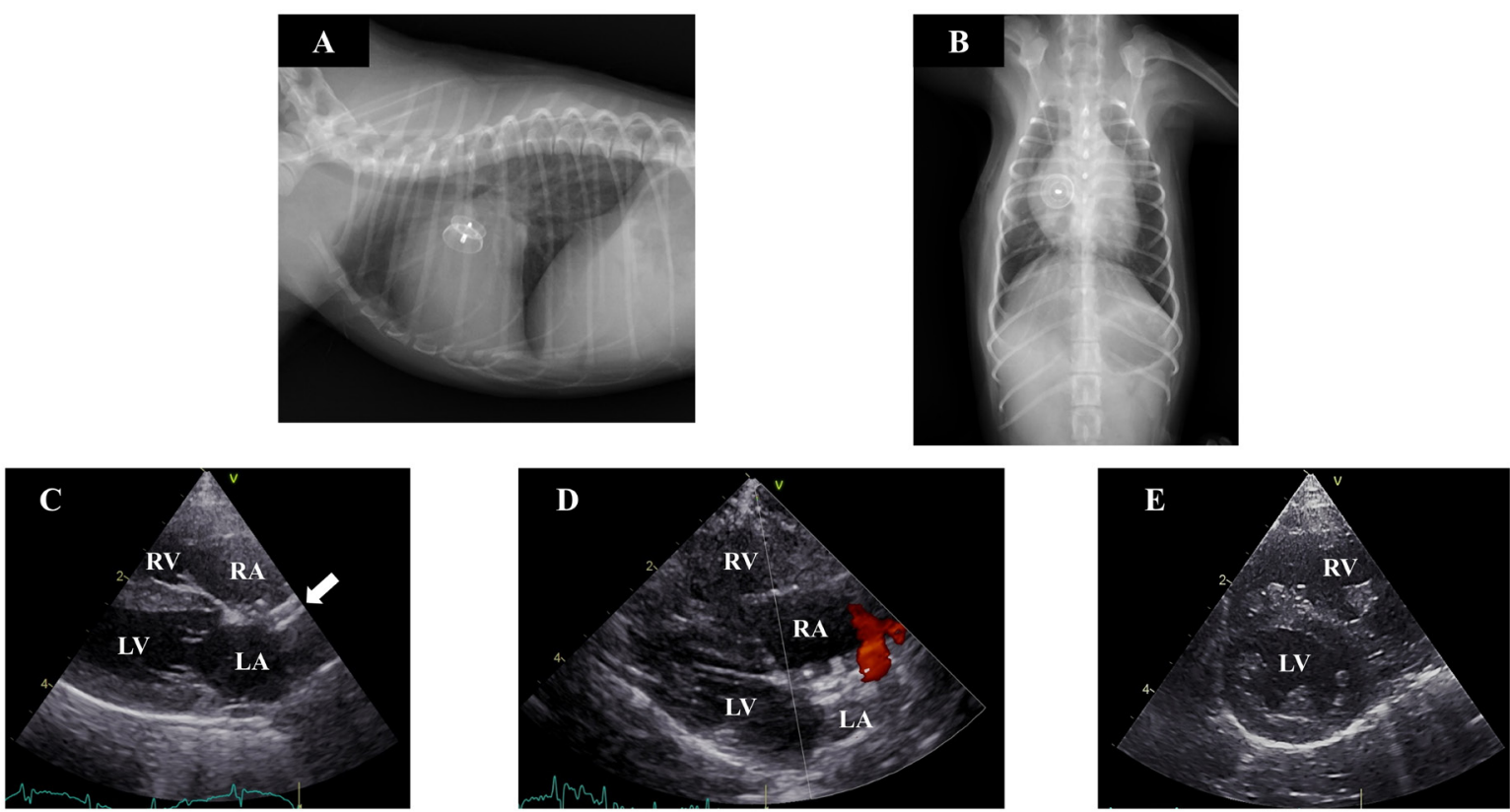

Fig. 2. Thoracic radiography and echocardiography 7 months after the operation. (a) and (b): In thoracic radiography, the device was clearly visible and there was no evidence of pulmonary overcirculation. (c)-(e): The device was appropriately positioned across the defect (solid arrow), and the right atrial and ventricular volume overload had almost resolved although mild residual shunting was observed. Ventricular septal flattening was also improved. (LA): left atrium; (LV): left ventricle; (RA): right atrium. 
midget breed dogs, as in the present case, the width of the atrial septum may be too thin for these methods, such methods may also be necessary to assess defect size.

The Japanese Circulation Society recommends that, in humans, any uncomplicated ASD with Qp/Qs $\geq 1.5$, defects $\geq 5 \mathrm{~mm}$ and $<38 \mathrm{~mm}$, or RV enlargement has been closed. It has also been reported that large dog breeds with defects $>12 \mathrm{~mm}$ may develop clinical signs (Gordon et al., 2009). However, no recommendations pertaining to small and midget dog breeds have been reported. This may be because the prevalence of ASD is reportedly higher in larger dog breeds including standard poodles and boxers (Chetboul et al., 2006; Guglielmini et al., 2002), and it is difficult to diagnose ASD since cardiac murmur is not always present (Chetboul et al., 2006). In the present case, ASD closure was performed because $\mathrm{Qp} / \mathrm{Qs} \geq 1.5$ and $\mathrm{RV}$ enlargement were evident. In the present case, an Amplatz canine duct occluder was used to close the ASD. Currently, the Amplatz septal occluder (ASO) is used for ASD closure in humans, and its use has also been reported in veterinary medicine (Gordon et al., 2009; 2010). However, in Japan, the ASO is not approved for use in veterinary medicine. Due to this, an Amplatz canine duct occluder was used, and the closure was successful.

This is the first report about the ASD closure in a midget breed dog. Further research involving a large sample size is warranted to evaluate the risks associated with the use of a hybrid approach for ASD closure in midget dogs.

\section{Conflict of interest}

The authors declare that there is no conflict of interest. Author's contributions

Conceptualization: Keisuke Sugimoto, Yohei Mochizuki, Masaki Kochi, and Takuma Aoki. Data curation: Keisuke Sugimoto. Formal analysis: Keisuke Sugimoto and Yohei Mochizuki. Investisgation: Keisuke Sugimoto, Yohei Mochizuki, Teppei Kanda, Akihiro Ohnishi, Masahiro Miyabe, and Yuko Wada. Methodology: Keisuke Sugimoto, Yohei Mochizuki, Teppei Kanda, Masaki Kochi, and Takuma Aoki. Supervision: Keisuke Sugimoto. Writing - original draft: Keisuke Sugimoto, Yohei Mochizuki, Teppei Kanda, Akihiro Ohnishi, Masahiro Miyabe, and Yuko Wada. Writing - review and editing: Keisuke Sugimoto.

\section{References}

Amin, Z., Cao, Q.L. and Hijazi, Z.M. 2008. Closure of muscular ventricular septal defects: Transcatheter and hybrid techniques. Catheter. Cardiovasc. Interv. $72,102-111$.

Brown, K.N. and Kanmanthareddy, A. 2019. Catheter management of atrial septal defect. In: StatPearls, Treasure Island (FL).

Carminati, M., Butera, G., Chessa, M., De Giovanni, J., Fisher, G., Gewillig, M., Peuster, M., Piechaud,
J.F., Santoro, G., Sievert, H., Spadoni, I., Walsh, K. and Investigators of the European, V.S.D.R. 2007. Transcatheter closure of congenital ventricular septal defects: results of the European Registry. Eur. Heart J. 28, 2361-2368.

Chetboul, V., Charles, V., Nicolle, A., Sampedrano, C.C., Gouni, V., Pouchelon, J.L. and Tissier, R. 2006. Retrospective study of 156 atrial septal defects in dogs and cats (2001-2005). J. Vet. Med. A Physiol. Pathol. Clin. Med. 53, 179-184.

Domenech, O. and Oliveira, P. 2013. Transoesophageal echocardiography in the dog. Vet. J. 198, 329-338.

Doshi, S.N., George, S., Kwok, C.S., Mechery, A., Mamas, M., Ludman, P.F., Townend, J.N. and Bhabra, M. 2018. A feasibility study of transaxillary TAVI with the lotus valve. Catheter. Cardiovasc. Interv. 92, 542-549.

Driver, K. and Mangrum, J.M. 2015. Hybrid approaches in atrial fibrillation ablation: why, where and who? J. Thorac. Dis. 7, 159-164.

Eyster, G.E., Anderson, L.K., Krehbeil, J.D., Whipple, R.D., Blanchard, G.L., Dickason, J., Braden, T.D., Bohonowych, R. and Harris, F. 1976. Surgical repair of atrial septal defect in a dog. J. Am. Vet. Med. Assoc. 169, 1081-1084.

Gordon, S.G., Miller, M.W., Roland, R.M., Saunders, A.B., Achen, S.E., Drourr, L.T. and Nelson, D.A. 2009. Transcatheter atrial septal defect closure with the Amplatzer atrial septal occluder in 13 dogs: short- and mid-term outcome. J. Vet. Intern. Med. 23, 995-1002.

Gordon, S.G., Nelson, D.A., Achen, S.E., Miller, M.M., Roland, R.M., Saunders, A.B. and Drourr, L.T. 2010. Open heart closure of an atrial septal defect by use of an atrial septal occluder in a dog. J. Am. Vet. Med. Assoc. 236, 434-439.

Guglielmini, C., Diana, A., Pietra, M. and Cipone, M. 2002. Atrial septal defect in five dogs. J. Small Anim. Pract. 43, 317-322.

Li, Q.Z., Cao, H., Chen, Q., Zhang, G.C., Chen, L.W. and Chen, D.Z. 2013. Balloon valvuloplasty through the right ventricle: another treatment of pulmonary atresia with intact ventricular septum. Ann. Thorac. Surg. 95, 1670-1674.

Ohnishi, Y. 2012. Hybrid approach combined with catheter intervention and surgical procedure for the hypoplastic left heart syndrome. Masui 61, 12161220 .

Pelosi, A., Anderson, L.K., Paugh, J., Robinson, S. and Eyster, G.E. 2013. Challenges of cardiopulmonary bypass-a review of the veterinary literature. Vet. Surg. 42, 119-136.

Sanders, R.A., Hogan, D.E., Green, H.W., Hoyer, M.H. and Puppel, D.A. 2005. Transcatheter closure of an atrial septal defect in a dog. J. Am. Vet. Med. Assoc. 227, 430-434. 
Schrope, D.P. 2015. Prevalence of congenital heart disease in 76,301 mixed-breed dogs and 57,025 mixed-breed cats. J. Vet. Cardiol. 17, 192-202.

Shelden, A., Wesselowski, S., Gordon, S.G. and Saunders, A.B. 2017. Transcatheter closure of a small atrial septal defect with an Amplatzer patent foramen ovale occluder in a working dog with cyanosis and exercise intolerance at high altitude. J. Vet. Cardiol. 19, 523-529.

Taniguchi, M., Akagi, T., Watanabe, N., Okamoto, Y., Nakagawa, K., Kijima, Y., Toh, N., Ohtsuki, S., Kusano, K. and Sano, S. 2009. Application of real-time three-dimensional transesophageal echocardiography using a matrix array probe for transcatheter closure of atrial septal defect. J. Am. Soc. Echocardiogr. 22, 1114-1120.

Uemura, A., Tanaka, R., Nakata, T., Goya, S., Shimada, K., Chantawong, P., Pitipipatukun, P., Hamabe, L. and Matsuura, K. 2018. Hybrid technique for closing an atrial septal defect using an Amplatz ${ }^{\mathbb{B}}$
Canine Duct Occluder in a small breed dog. Aust. Vet. Pract. 48(1), 19-21.

van den Bosch, A.E., Ten Harkel, D.J., McGhie, J.S., Roos-Hesselink, J.W., Simoons, M.L., Bogers, A.J. and Meijboom, F.J. 2006. Characterization of atrial septal defect assessed by real-time 3-dimensional echocardiography. J. Am. Soc. Echocardiogr. 19, 815-821.

Zanchetta, M., Onorato, E., Rigatelli, G., Pedon, L., Zennaro, M., Carrozza, A. and Maiolino, P. 2003. Intracardiac echocardiography-guided transcatheter closure of secundum atrial septal defect: a new efficient device selection method. J. Am. Coll. Cardiol. 42, 1677-1682.

Zani, A., Becchetti, E., Chimenti, T., Daddi, V., Zani, M., Miotti, C. and Bussadori, C. 2016. Use of an amplatz canine ductal occluder (ACDO) device to close an acquired aortopulmonary fistula with a hybrid approach in a dog. J. Vet. Cardiol. 18, 377384. 